\title{
UNIVERSALITY OF THE LATTICE OF TRANSFORMATION MONOIDS
}

\author{
MICHAEL PINSKER AND SAHARON SHELAH
}

(Communicated by Julia Knight)

\begin{abstract}
The set of all transformation monoids on a fixed set of infinite cardinality $\lambda$, equipped with the order of inclusion, forms a complete algebraic lattice $\operatorname{Mon}(\lambda)$ with $2^{\lambda}$ compact elements. We show that this lattice is universal with respect to closed sublattices; i.e., the closed sublattices of $\operatorname{Mon}(\lambda)$ are, up to isomorphism, precisely the complete algebraic lattices with at most $2^{\lambda}$ compact elements.
\end{abstract}

\section{Definitions And the Result}

Fix an infinite set; for the sake of simpler notation, we identify the set with its cardinality $\lambda$. By a transformation monoid on $\lambda$ we mean a subset of $\lambda^{\lambda}$ which is closed under composition and which contains the identity function. The set of transformation monoids acting on $\lambda$, ordered by inclusion, forms a complete lattice $\operatorname{Mon}(\lambda)$ in which the meet of a set of monoids is simply their intersection. This lattice is algebraic; i.e., every element is a join of compact elements. An element $a$ in a complete lattice $\mathfrak{L}=(L, \vee, \wedge)$ is called compact iff whenever $A \subseteq L$ and $a \leq \bigvee A$, there is a finite $A^{\prime} \subseteq A$ such that $a \leq \bigvee A^{\prime}$. In the case of $\operatorname{Mon}(\lambda)$, the compact elements are precisely the finitely generated monoids, i.e., those monoids which contain a finite set of functions such that every function of the monoid can be composed from functions of this finite set. Consequently, the number of compact elements of $\operatorname{Mon}(\lambda)$ equals $2^{\lambda}$.

It is well-known and not hard to see that for any cardinal $\kappa$, the algebraic lattices with at most $\kappa$ compact elements are, up to isomorphism, precisely the subalgebra lattices of algebras whose domains have $\kappa$ elements ([BF48]; see also Theorem 48 in the textbook Grä03]). For example, $\operatorname{Mon}(\lambda)$ is the subalgebra lattice of the algebra which has domain $\lambda^{\lambda}$, a binary operation which is the function composition on $\lambda^{\lambda}$, as well as a constant operation whose value is the identity function on $\lambda$.

Received by the editors June 24, 2011 and, in revised form, September 3, 2011, September 12, 2011, and November 24, 2011.

2010 Mathematics Subject Classification. Primary 06B15; Secondary 06B23, 20M20.

Key words and phrases. Algebraic lattice, transformation monoid, submonoid, closed sublattice.

The research of the first author was supported by an APART fellowship of the Austrian Academy of Sciences.

The research of the second author was supported by German-Israeli Foundation for Scientific Research \& Development Grant No. 963-98.6/2007.

The authors would like to thank an anonymous referee for valuable comments which led to significant improvements in the presentation of the paper. 
Let $\mathfrak{K}$ and $\mathfrak{L}$ be complete lattices such that the domain of $\mathfrak{L}$ is contained in the domain of $\mathfrak{K}$. Then $\mathfrak{L}$ is called a complete sublattice of $\mathfrak{K}$ iff all joins and meets in $\mathfrak{L}$ equal the corresponding joins and meets in $\mathfrak{K}$. In most of the literature where the notion is mentioned and, in particular, in the widely used textbook Grä03, this includes empty joins and meets, and so in this definition the respective largest and smallest elements of $\mathfrak{L}$ and $\mathfrak{K}$ must coincide. When the condition of "complete sublattice" holds for all non-empty joins and meets, we say that $\mathfrak{L}$ is a closed sublattice of $\mathfrak{K}$; the difference here is that the respective largest and smallest elements of $\mathfrak{L}$ and $\mathfrak{K}$ need not coincide. In other words, $\mathfrak{L}$ is a closed sublattice of $\mathfrak{K}$ if and only if it is a complete sublattice of a closed interval of $\mathfrak{K}$.

Now let $\mathfrak{K}$ be a complete algebraic lattice and $\mathfrak{L}$ be a closed sublattice of $\mathfrak{K}$. Then it is a well-known fact that $\mathfrak{L}$ is algebraic as well and that the number of compact elements of $\mathfrak{L}$ equals at most the corresponding number for $\mathfrak{K}$. For the comfort of the reader, let us sketch the argument showing this. Denote for every compact element $x$ of $\mathfrak{K}$ which is below some element of $\mathfrak{L}$ the smallest element of $\mathfrak{L}$ which is above $x$ by $x^{\mathfrak{L}}$. Then it is not hard to see that the compact elements of $\mathfrak{L}$ are precisely the elements of the form $x^{\mathfrak{L}}$. Hence, the mapping that sends every $x$ as above to $x^{\mathfrak{L}}$ shows that $\mathfrak{L}$ does not possess more compact elements than $\mathfrak{K}$, and it also follows easily from the above that $\mathfrak{L}$ is algebraic.

By this observation, any closed sublattice of $\operatorname{Mon}(\lambda)$ is algebraic and has at most $2^{\lambda}$ compact elements. In this paper, we prove the converse of this fact. This had been stated as an open problem in [GP08, Problem C]. We remark that it is clear from the context in GP08 that the word "subinterval" in the formulation of Problem $\mathrm{C}$ is an error; it is Problem B which asks about subintervals. We also note that while in GP08] the authors write "complete sublattice," they confirmed upon inquiry that they really meant "closed sublattice," although they consider the question about complete sublattices interesting as well.

Theorem 1.1. $\operatorname{Mon}(\lambda)$ is universal for complete algebraic lattices with at most $2^{\lambda}$ compact elements with respect to closed sublattices; i.e., the closed sublattices of $\operatorname{Mon}(\lambda)$ are, up to isomorphism, precisely the complete algebraic lattices with at most $2^{\lambda}$ compact elements.

We remark that it follows from our proof that if $\mathfrak{L}$ is an algebraic lattice with at most $2^{\lambda}$ compact elements, then it is even isomorphic to a closed sublattice of $\operatorname{Mon}(\lambda)$ via an isomorphism which preserves the smallest element (but not the largest, in which case we would obtain a complete sublattice).

\section{RELATED WORK AND POSSIBLE EXTENSIONS}

2.1. Cardinality questions and non-closed sublattices. It has been known for a long time that every (not necessarily complete) lattice $\mathfrak{L}$ is isomorphic to a sublattice of the lattice of subgroups of a group Whi46. Hence, viewing the group as a monoid, it follows that every lattice is isomorphic to a sublattice of the lattice of submonoids of a monoid $\mathfrak{M}$. Strengthening of the latter statement was obtained in Rep96, where it was shown that one can impose a variety of different additional properties on the monoid $\mathfrak{M}$. In these theorems, one cannot simply replace "sublattice" by "closed sublattice", since the lattice of subalgebras of an algebra is algebraic, and hence, by our discussion above, closed sublattices must share the same property. 
There are lattices $\mathfrak{L}$ of size $\kappa$ for which the corresponding monoid $\mathfrak{M}$ must have size at least $\kappa$ as well. For example, it is easy to see that this is the case when $\mathfrak{L}$ is the lattice on $\kappa$ induced by the natural order of $\kappa$. When $\mathfrak{L}$ is complete, algebraic, and has $\kappa$ compact elements, the monoid $\mathfrak{M}$ will generally have to have size at least $\kappa$, although $\mathfrak{L}$ itself might very well have size $2^{\kappa}$. This improvement on the obvious general lower bound for the cardinality of $\mathfrak{M}$ compared to arbitrary lattices is due to the fact that the structure of $\mathfrak{L}$ is already determined by the structure of the join-semilattice of its compact elements. A given abstract monoid $\mathfrak{M}$ can then in turn be realized as a transformation monoid by letting it act on itself; hence, it will act on a set of size $\kappa$. In our case, this would yield an embedding into $\operatorname{Mon}\left(2^{\lambda}\right)$; the difficulty of our theorem is to find $\mathfrak{L}$ as a closed sublattice of the optimal $\operatorname{Mon}(\lambda)$.

2.2. Closed sublattices of related algebraic lattices. A clone on $\lambda$ is a set of finitary operations on $\lambda$ which is closed under composition and which contains all finitary projections; in other words, it is a set of finitary operations closed under building of terms (without constants). The set of all clones on $\lambda$, ordered by inclusion, also forms a complete algebraic lattice $\mathrm{Cl}(\lambda)$ with $2^{\lambda}$ compact elements into which $\operatorname{Mon}(\lambda)$ embeds naturally, since a transformation monoid can be viewed as a clone, all of whose operations depend on at most one variable. Universality of $\mathrm{Cl}(\lambda)$ for complete algebraic lattices with at most $2^{\lambda}$ compact elements with respect to closed sublattices has been shown in Pin07. (the author of Pin07] writes "complete sublattices" but really means - and proves - "closed sublattices"); our result is a strengthening of this result.

Observe that similarly to transformation monoids and clones, the set of permutation groups on $\lambda$ forms a complete algebraic lattice $\operatorname{Gr}(\lambda)$ with respect to inclusion. By virtue of the identity embedding, $\operatorname{Gr}(\lambda)$ is a complete sublattice of $\operatorname{Mon}(\lambda)$. We do not know the following.

Problem 2.1. Is every complete algebraic lattice with at most $2^{\lambda}$ compact elements a closed sublattice of $\operatorname{Gr}(\lambda)$ ?

In this context it is worthwhile mentioning that in our proof of Theorem 1.1. we exclusively use monoids which only contain permutations. In other words, we construct for every complete algebraic lattice with at most $2^{\lambda}$ compact elements a closed sublattice of the interval of $\operatorname{Mon}(\lambda)$ consisting of those monoids on $\lambda$ which are subsets of the symmetric group on $\lambda$. However, our "permutation monoids" are themselves not groups, and in fact they never contain the inverse of any of their permutations (except the identity) - adding inverses would collapse the construction.

A related problem is when lattices appear as intervals of $\operatorname{Gr}(\lambda), \operatorname{Mon}(\lambda)$, and $\mathrm{Cl}(\lambda)$. This remains open: for the latter two lattices this question has been posed as an open problem in GP08 (Problems B and A, respectively). By a deep theorem due to Tơma [Ti̊m89], every complete algebraic lattice with $\lambda$ compact elements is isomorphic to an interval of the subgroup lattice of a group of size $\lambda$. From this it follows only that $\operatorname{Gr}(\lambda)$ contains all complete algebraic lattices with at most $\lambda$ compact elements as intervals. Proving that $\operatorname{Gr}(\lambda)$ contains all complete algebraic lattices with at most $2^{\lambda}$ compact elements as intervals would be a common strengthening of Tůma's result and a positive answer to Problem 2.1 . 


\section{Proof of the theorem}

3.1. Independent composition engines. For a cardinal $\kappa$ and a natural number $n \geq 1$, we write $\Lambda_{\kappa}^{n}:=\kappa^{n} \times 2^{n}$. We set $\Lambda_{\kappa}:=\bigcup_{n>1} \Lambda_{\kappa}^{n}$. For sequences $p, q$, we write $p \triangleleft q$ if $p$ is a non-empty initial segment of $q$ (we consider $q$ to be an initial segment of itself). For $(\eta, \phi)$ and $\left(\eta^{\prime}, \phi^{\prime}\right)$ in $\Lambda_{\kappa}$, we also write $(\eta, \phi) \triangleleft\left(\eta^{\prime}, \phi^{\prime}\right)$ if $\eta \triangleleft \eta^{\prime}$ and $\phi \triangleleft \phi^{\prime}$. If $p$ is a sequence and $r$ a set, then $p * r$ denotes the extension of $p$ by the element $r$. We write $\langle r\rangle$ for the one-element sequence containing only $r$.

A sequence $P$ of elements of $\Lambda_{\kappa}$ is reduced iff it does not contain both $(\eta * \alpha, \phi * 0)$ and $(\eta * \alpha, \phi * 1)$ for any $(\eta, \phi) \in \Lambda_{\kappa}$ and $\alpha \in \kappa$. We call two sequences $P, Q$ equivalent iff $P$ can be transformed into $Q$ by permuting its elements.

For a set $W$ and a cardinal $\kappa$, a $\kappa$-branching independent composition engine $(\kappa-I C E)$ on $W$ is an indexed set $\left\{f_{(\eta, \phi)}:(\eta, \phi) \in \Lambda_{\kappa}\right\}$ of permutations on $W$ satisfying all of the following:

(i) (Composition) For all $(\eta, \phi) \in \Lambda_{\kappa}$ and for all $\alpha \in \kappa$, we have $f_{(\eta, \phi)}=$ $f_{(\eta * \alpha, \phi * 0)} \circ f_{(\eta * \alpha, \phi * 1)}$

(ii) (Commutativity) For all $a, b \in \Lambda_{\kappa}$, we have that $f_{a} \circ f_{b}=f_{b} \circ f_{a}$.

(iii) (Independence) Whenever $P=\left(p_{1}, \ldots, p_{n}\right)$ and $Q=\left(q_{1}, \ldots, q_{m}\right) \subseteq \Lambda_{\kappa}$ are inequivalent reduced sequences, $t_{P}:=f_{p_{1}} \circ \cdots \circ f_{p_{n}}$ and $t_{Q}:=f_{q_{1}} \circ \cdots \circ f_{q_{m}}$ are not equal.

Note that by the commutativity of the system, the order of the elements of the sequences $P$ and $Q$ in condition (iii) is not of importance.

Lemma 3.1. There exists a $2^{\lambda}-I C E$ on $\lambda$.

Proof. We show that there exists a $2^{\lambda}$-ICE on $W:=\lambda \times \mathbb{Z}$. Let

$$
\mathcal{A}:=\left\{A_{(\eta, \phi)}:(\eta, \phi) \in \Lambda_{2^{\lambda}} \text { and the last entry of } \phi \text { equals } 0\right\}
$$

be an independent family of subsets of $\lambda$; i.e., any non-trivial finite Boolean combination of these sets is non-empty (see, for example, [Jec03, Lemma 7.7] for a proof of the existence of such a family). For all $(\eta, \phi) \in \Lambda_{2^{\lambda}}$, set $\# A_{(\eta, \phi)}$ equal to $A_{(\eta, \phi)}$ if the last entry of $\phi$ equals 0 , and $\lambda \backslash A_{\left(\eta, \phi^{\prime}\right)}$ otherwise, where $\phi^{\prime}$ is obtained from $\phi$ by changing the last entry to 0 . Now define $B_{(\eta, \phi)}:=\bigcap_{s \triangleleft(\eta, \phi)} \# A_{s}$ for all $(\eta, \phi) \in \Lambda_{2^{\lambda}}$.

We will define the $2^{\lambda}$-ICE by means of the family $\left\{B_{(\eta, \phi)}:(\eta, \phi) \in \Lambda_{2^{\lambda}}\right\}$ as follows. For all $(\eta, \phi) \in \Lambda_{2^{\lambda}}$ and all $(\alpha, i) \in W$, we set

$$
f_{(\eta, \phi)}(\alpha, i)= \begin{cases}(\alpha, i+1), & \text { if } \alpha \in B_{(\eta, \phi)} \\ (\alpha, i), & \text { otherwise }\end{cases}
$$

We claim that this defines a $2^{\lambda}$-ICE on $W$. Clearly, (ii) of the definition is satisfied. Property (i) is a direct consequence of the fact that for all $(\eta, \phi) \in \Lambda_{2^{\lambda}}$ and all $\alpha<\lambda, B_{(\eta, \phi)}$ is the disjoint union of $B_{(\eta * \alpha, \phi * 0)}$ and $B_{(\eta * \alpha, \phi * 1)}$.

To see (iii), let $P$ and $Q$ be reduced and inequivalent. We first claim that we can assume that $P$ and $Q$ have no entries in common. So say that some $(\eta, \phi)$ of $\Lambda_{2^{\lambda}}$ occurs in both $P$ and $Q$. Then, since $t_{P}=t_{Q}$ if and only if $f_{(\eta, \phi)}^{-1} \circ t_{P}=f_{(\eta, \phi)}^{-1} \circ t_{Q}$, proving that $t_{P}$ is not equal to $t_{Q}$ is the same as proving that $t_{P^{\prime}}$ is not equal to $t_{Q^{\prime}}$, where for $X \in\{P, Q\}$ we write $X^{\prime}$ for the sequence obtained from $X$ by removing 
one occurrence of $(\eta, \phi)$. Observe that as subsequences of $P$ and $Q$ respectively, $P^{\prime}$ and $Q^{\prime}$ are still reduced. Repeating this process, we may indeed assume that $P$ and $Q$ have no common entries. Now to prove that $t_{P} \neq t_{Q}$, let $(\eta, \phi)$ be an element of $\Lambda_{2^{\lambda}}$ which appears either in $P$ or in $Q$ and which has the property that $\left(\eta^{\prime}, \phi^{\prime}\right)=(\eta, \phi)$ for all pairs $\left(\eta^{\prime}, \phi^{\prime}\right)$ which appear in $P$ or $Q$ and for which $\left(\eta^{\prime}, \phi^{\prime}\right) \triangleleft(\eta, \phi)$. Say without loss of generality that $(\eta, \phi)$ appears in $P$. Let $A$ be the union of all $B_{q}$ for which $q$ appears in $Q$. Then it follows from the independence of the family $\mathcal{A}$, from the fact that $Q$ is reduced, and from the fact that $Q$ contains no $q$ with $q \triangleleft(\eta, \phi)$ that $B_{(\eta, \phi)}$ is not contained in the union of all \# $A_{q}$ for $q$ appearing in $Q$, and thus $B_{(\eta, \phi)} \backslash A$ is non-empty. Let $\alpha$ be an element of the latter set. Then $t_{P}(\alpha, 0)=(\alpha, k)$ for some $k>0$, whereas $t_{Q}(\alpha, 0)=(\alpha, 0)$. Hence, $t_{P} \neq t_{Q}$.

3.2. From lattices to monoids. For a $\kappa$-ICE $\left\{f_{(\eta, \phi)}:(\eta, \phi) \in \Lambda_{\kappa}\right\}$ on $W$ and a subset $S$ of $\Lambda_{\kappa}$, we set $F(S)$ to be the monoid generated by the functions with index in $S$, i.e., the smallest monoid of functions from $W$ to $W$ which contains all the functions with index in $S$. Another way to put it is that $F(S)$ contains precisely the composites of functions with index in $S$ as well as the identity function on $W$.

In the following, fix a $2^{\lambda}$-ICE $\left\{f_{(\eta, \phi)}:(\eta, \phi) \in \Lambda_{2^{\lambda}}\right\}$ on $\lambda$. Let $\mathfrak{L}=(L, \vee, \wedge)$ be any complete algebraic lattice with $2^{\lambda}$ compact elements. Let $C \subseteq L$ be the set of compact elements of $\mathfrak{L}$ excluding the smallest element. Enumerate $C$, possibly with repetitions, by $\left\{c_{(\eta, \phi)}:(\eta, \phi) \in \Lambda_{2^{\lambda}}\right\}$ and in such a way that the following hold:

(1) Every element of $C$ is equal to $c_{(\langle\alpha\rangle,\langle 0\rangle)}$ for some $\alpha<2^{\lambda}$.

(2) For all $(\eta, \phi) \in \Lambda_{2^{\lambda}}$ and all $\alpha \in 2^{\lambda}$, we have $c_{(\eta, \phi)} \leq c_{(\eta * \alpha, \phi * 0)} \vee c_{(\eta * \alpha, \phi * 1)}$.

(3) For all $(\eta, \phi) \in \Lambda_{2^{\lambda}}$ and all $d, d^{\prime} \in C$ with $c_{(\eta, \phi)} \leq d \vee d^{\prime}$, there exists $\alpha<2^{\lambda}$ such that $d=c_{(\eta * \alpha, \phi * 0)}$ and $d^{\prime}=c_{(\eta * \alpha, \phi * 1)}$.

Consider the semilattice $(C, \vee)$ of compact elements of $\mathfrak{L}$ without the smallest element. An ideal of $(C, \vee)$ is a possibly empty subset of $C$ which is downward closed and closed under finite joins. It is a straightforward consequence of the fact that $\mathfrak{L}$ is algebraic that $\mathfrak{L}$ is isomorphic to the lattice of ideals of $(C, \vee)$ (the statement is equivalent to Theorem 42 in Grä03]). The meet $\bigwedge_{u \in U} I_{u}$ of a nonempty set of ideals $\left\{I_{u}: u \in U\right\}$ in this lattice is just their intersection; their join $\bigvee_{u \in U} I_{u}$ is the smallest ideal containing all $I_{u}$, that is, the set of all elements $c$ of $C$ for which there exist $c_{1}, \ldots, c_{n} \in \bigcup_{u \in U} I_{u}$ such that $c \leq c_{1} \vee \cdots \vee c_{n}$.

To every ideal $I \subseteq C$, assign the sets $S(I):=\left\{(\eta, \phi) \in \Lambda_{2^{\lambda}}: c_{(\eta, \phi)} \in I\right\}$ and $F(I):=F(S(I))$.

Lemma 3.2. If $\left\{I_{u}: u \in U\right\}$ is a non-empty set of ideals of $(C, \vee)$, then $\bigvee_{u \in U} F\left(I_{u}\right)$ $=F\left(\bigvee_{u \in U} I_{u}\right)$.

Proof. The inclusion $\subseteq$ is trivial. For the other direction, it is enough to show that if $c_{(\eta, \phi)}$ is an element of $\bigvee_{u \in U} I_{u}$, then $f_{(\eta, \phi)}$ is an element of $\bigvee_{u \in U} F\left(I_{u}\right)$. There exist $c_{\left(\eta_{1}, \phi_{1}\right)}, \ldots, c_{\left(\eta_{n}, \phi_{n}\right)} \in \bigcup_{u \in U} I_{u}$ such that $c_{(\eta, \phi)} \leq c_{\left(\eta_{1}, \phi_{1}\right)} \vee \cdots \vee c_{\left(\eta_{n}, \phi_{n}\right)}$. We use induction over $n$. If $n=1$, then $c_{(\eta, \phi)} \leq c_{\left(\eta_{1}, \phi_{1}\right)} \in I_{u}$ for some $u \in U$, so $c_{(\eta, \phi)} \in I_{u}$. Hence, $f_{(\eta, \phi)} \in F\left(I_{u}\right)$, and we are done. In the induction step, suppose the claim 
holds for all $1 \leq k<n$. Set $d:=c_{\left(\eta_{1}, \phi_{1}\right)} \vee \cdots \vee c_{\left(\eta_{n-1}, \phi_{n-1}\right)}$ and $d^{\prime}:=c_{\left(\eta_{n}, \phi_{n}\right)}$. Since $c_{(\eta, \phi)} \leq d \vee d^{\prime}$, there exist $\alpha<2^{\lambda}$ such that $\left(d, d^{\prime}\right)=\left(c_{(\eta * \alpha, \phi * 0)}, c_{(\eta * \alpha, \phi * 1)}\right)$, by property (3) of our enumeration. By the induction hypothesis, we have $f_{d}, f_{d^{\prime}} \in$ $\bigvee_{u \in U} F\left(I_{u}\right)$. Since $f_{(\eta, \phi)}=f_{(\eta * \alpha, \phi * 0)} \circ f_{(\eta * \alpha, \phi * 1)}$, we get that $f_{(\eta, \phi)} \in \bigvee_{u \in U} F\left(I_{u}\right)$ as well, proving the lemma.

Lemma 3.3. If $\left\{I_{u}: u \in U\right\}$ is a non-empty set of ideals of $(C, \vee)$, then $\bigcap_{u \in U} F\left(I_{u}\right)$ $=F\left(\bigcap_{u \in U} I_{u}\right)$.

Proof. This time, the inclusion $\supseteq$ is trivial. For the other direction, let $t \in$ $\bigcap_{u \in U} F\left(I_{u}\right)$, and assume that $t$ is not the identity function. Then there is a unique reduced set $P$ such that $t=t_{P}$, by property (iii) of an independent composition engine. Now let $u \in U$ be arbitrary. Then there exists a sequence $Q$ in $S\left(I_{u}\right)$ such that $t=t_{Q}$. By subsequently replacing two entries $(\eta * \alpha, \phi * 0),(\eta * \alpha, \phi * 1)$ in $Q$ by $(\eta, \phi)$, we obtain a reduced sequence $Q^{\prime}$ which still satisfies $t=t_{Q^{\prime}}$. Since $I_{u}$ is closed under joins, and by property (2) of our enumeration of the compact elements, all entries of $Q^{\prime}$ are still elements of $S\left(I_{u}\right)$. By the independence property, $P$ and $Q^{\prime}$ are equivalent, and hence $P$ is a sequence in $S\left(I_{u}\right)$. But $u$ was arbitrary, so $P$ is a sequence in $\bigcap_{u \in U} S\left(I_{u}\right)$. This proves $t \in F\left(\bigcap_{u \in U} I_{u}\right)$.

Lemma 3.4. If $I, J$ are distinct ideals of $(C, \vee)$, then $F(I) \neq F(J)$.

Proof. Assume without loss of generality that $I \backslash J$ is non-empty. Then $S(I) \backslash S(J)$ is non-empty as well; let $(\eta, \phi)$ be an element therein. If we had $f_{(\eta, \phi)} \in F(J)$, then there would exist a sequence $Q$ in $S(J)$ such that $f_{(\eta, \phi)}=t_{Q}$. As in the proof of the preceding lemma, we could moreover assume that $Q$ is reduced. But then by the independence property, the sequence $Q$ would be equivalent with the sequence $\langle(\eta, \phi)\rangle$, implying $(\eta, \phi) \in S(J)$, a contradiction. So $f_{(\eta, \phi)} \notin F(J)$, but $f_{(\eta, \phi)} \in F(I)$, and we are done.

It follows from the above that the assignment $I \mapsto F(I)$ is an injective mapping from the lattice of ideals of $(C, \vee)$ to $\operatorname{Mon}(\lambda)$ which preserves arbitrary non-empty joins and meets, proving our theorem. Moreover, under this mapping the smallest element of $\mathfrak{L}$, represented by the empty ideal in $(C, \vee)$, is sent to the monoid which contains only the identity function, and hence to the smallest element of $\operatorname{Mon}(\lambda)$. This justifies the remark after the theorem.

\section{REFERENCES}

[BF48] Garrett Birkhoff and Orrin Frink, Jr., Representations of lattices by sets, Transactions of the American Mathematical Society 64 (1948), 299-316. MR0027263 (10:279g)

[GP08] Martin Goldstern and Michael Pinsker, A survey of clones on infinite sets, Algebra Universalis 59 (2008), 365-403. MR2470587 (2009j:08007)

[Grä03] George Grätzer, General lattice theory, Birkhäuser Verlag, Basel, 2003, reprint of the 1998 second edition. MR.1670580 (2000b:06001)

[Jec03] Thomas Jech, Set theory, Springer Monographs in Mathematics, Springer-Verlag, Berlin, 2003. The third millennium edition, revised and expanded. MR.1940513 (2004g:03071)

[Pin07] Michael Pinsker, Algebraic lattices are complete sublattices of the clone lattice on an infinite set, Fundamenta Mathematicae 195 (2007), no. 1, 1-10. MR2314073 (2008g:08002) 
[Rep96] Vladimir B. Repnitskiı̌, On representation of lattices by lattices of subsemigroups, Russian Mathematics 40 (1996), no. 1, 55-64 (translation of Russian original). MR1424151 (97k:06017)

[Tům89] Jiří Tůma, Intervals in subgroup lattices of infinite groups, Journal of Algebra $\mathbf{1 2 5}$ (1989), no. 2, 367-399. MR.1018952 (91e:20020)

[Whi46] Philip M. Whitman, Lattices, equivalence relations and subgroups, Bulletin of the American Mathematical Society 52 (1946), 507-522. MR0016750(8:62b)

Équipe de Logique Mathématique, Université Diderot - Paris 7, UFR de MathémaTiQues, 75205 Paris Cedex 13, France

E-mail address: marula@gmx.at

$U R L:$ http://dmg.tuwien.ac.at/pinsker/

Institute of Mathematics, The Hebrew University of Jerusalem, 91904 Jerusalem, Israel - And - Department of Mathematics, Rutgers University, New Brunswick, New JERSEY 08854

E-mail address: shelah@math.huji.ac.il

URL: http://shelah.logic.at 